\title{
Elotuzumab spares dendritic cell integrity and functionality
}

\author{
Sebastian Schlaweck ${ }^{1}\left(\mathbb{D} \cdot\right.$ Leon Strauss $^{1} \cdot$ Solveig Daecke $^{1} \cdot$ Peter Brossart $^{1} \cdot$ Annkristin Heine $^{1}(\mathbb{0}$
}

Received: 28 January 2021 / Accepted: 15 February 2021 / Published online: 2 March 2021

(c) The Author(s) 2021

Keywords Elotuzumab $\cdot$ Multiple myeloma $\cdot$ Dendritic cells $\cdot \mathrm{T}$ cell activation

\section{To the editor}

The armamentarium of proteasome inhibitors and IMiDs for Multiple Myeloma (MM) therapy was recently extended to monoclonal antibodies, which now are even investigated or are already approved as therapeutic option in first-line treatment (Mateos et al. 2018). Among these, the CD38 antibody daratumumab as well as the SLAMF7 antibody elotuzumab have shown remarkable results in MM therapy providing new treatment options. The results from the ELOQUENT2 and ELOQUENT3 study, both investigating the impact of adding elotuzumab to a back bone consisting of immunomodulatory drugs (IMiDs) and steroids extended the therapeutic options for patients suffering from relapsed or refractory MM.

Recently, the results from the ELOQUENT3 study have been supported by real-world data, underlining again that neutropenia and pneumonia are serious side effects of this therapeutic regime (Hose et al. 2021).

MM patients have been described to be per se sevenfold more susceptible to develop infections compared to population controls (Girmenia et al. 2019). Thus, detailed knowledge of potential immunosuppressive side effects of anti-Myeloma therapy is mandatory. Regarding elotuzumab, data about susceptibility for infections is inconsistent. The ELOQUENT3 study did not report any increased risk for infections for patients receiving elotuzumab together with pomalidomide and dexamethasone (Dimopoulos et al. 2018). In contrast, the final results from the ELOQUENT2 trial

Sebastian Schlaweck and Leon Strauss contributed equally to this work.

Annkristin Heine

Annkristin.Heine@ukbonn.de

1 Medical Clinic III for Oncology, Hematology, Immune-Oncology and Rheumatology, University Hospital Bonn, Venusberg Campus 1, 53127 Bonn, Germany acknowledged higher infection rates for the experimental group receiving elotuzumab in combination with lenalidomide and dexamethasone (ERd) compared to patients treated with lenalidomide and dexamethasone $(\mathrm{Rd})$ alone (Dimopoulos et al. 2020). In detail, elotuzumab-treated patients were prone to infections in general ( $84 \%$ in ERd vs $75 \%$ in Rd) and to pneumonia (22\% in ERd vs. $16 \%$ in Rd) as well as Herpes zoster reactivation (7\% in ERd vs. $2 \%$ in Rd) (Dimopoulos et al. 2020). Data elucidating whether the elimination of benign antibody-producing plasma cells, drug-induced neutropenia or additional off target effects contribute to this observation is scarce. However, it is already known that targeting SLAMF7, which is overexpressed on the surface of malignant plasma cells (Hsi et al. 2008), has not only direct effects on myeloma cells but also activates NK cells (Campbell et al. 2018), indicating that elotuzumab does not solely target MM cells, but also other immune cells. Elotuzumab triggers the antibody-dependent cellular cytotoxicity by NK cells and macrophages. Moreover, it promotes co-stimulatory pathways in NK cells myeloma-dependently and -independently by binding SLAMF7 on the NK cell surface (Campbell et al. 2018).

Dendritic cells (DCs) are essential in the induction of appropriate immune responses as they link the adaptive and innate immune system (Carbone et al. 1998), but, commonly used anti-myeloma drugs interfere with DCs. In detail, proteasome inhibitors and IMiDs suppress DC functionality (Nencioni et al. 2006a, b; Yamamoto et al. 2019) as well as daratumumab depletes plasmacytoid DCs and modulates PD-L1 expression on antigen presenting cells (Stocker et al. 2020), while it upregulates CD80 and CD86 on monocytes (Viola et al. 2020). Given the increased infection rate observed in MM patients, we wondered whether elotuzumab modulates DC phenotype, function and their ability to induce $\mathrm{T}$ cell responses as well.

To investigate the effects of elotuzumab, we isolated peripheral blood mononuclear cells (PBMCs) from buffy 
A

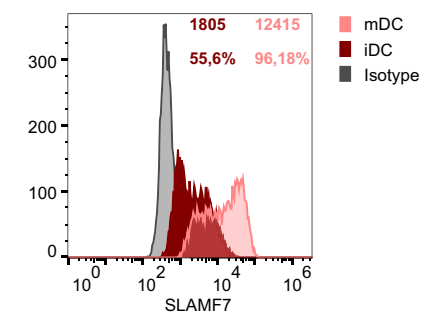

B

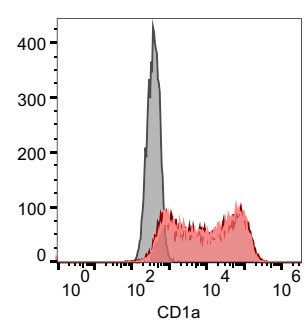

C

Elotuzumab treated mDCs untreated mDCs

Isotype

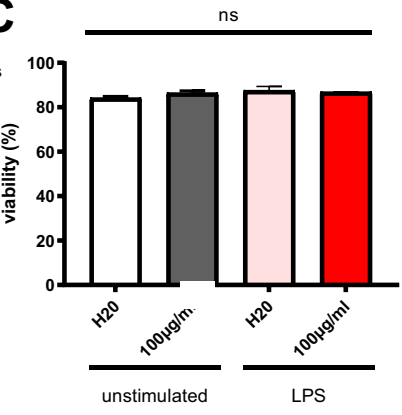

D

untreated

untreated

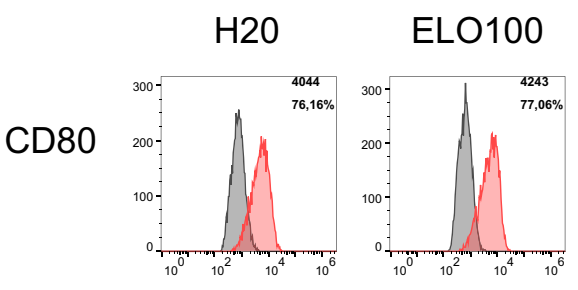

$\mathrm{H} 2 \mathrm{O}$

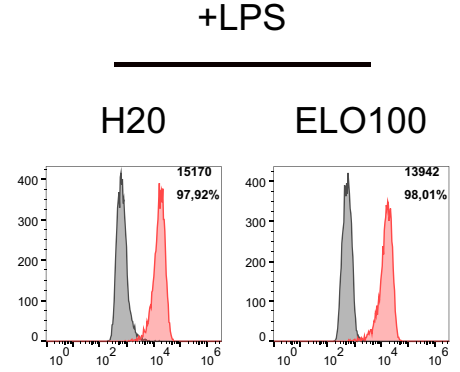

+ LPS
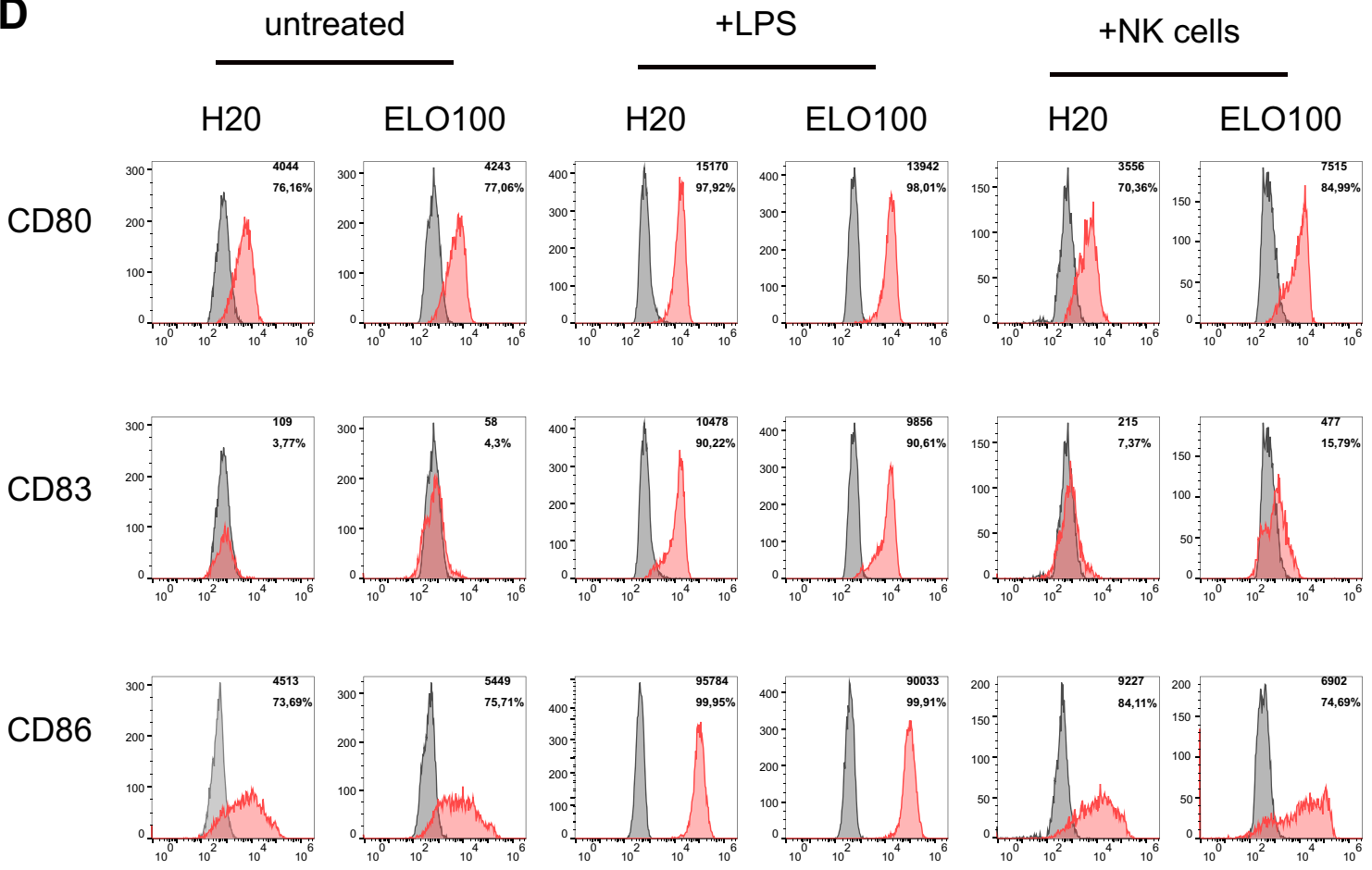

E
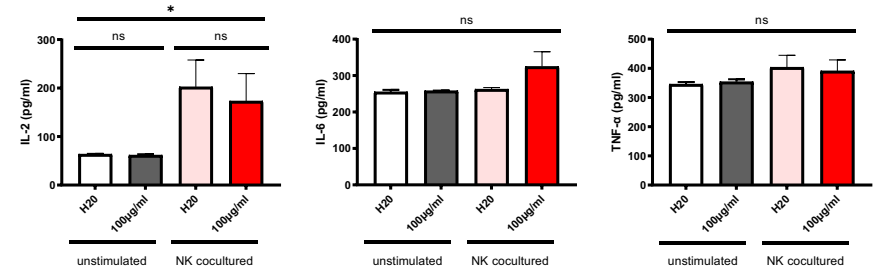

$\mathbf{F}$
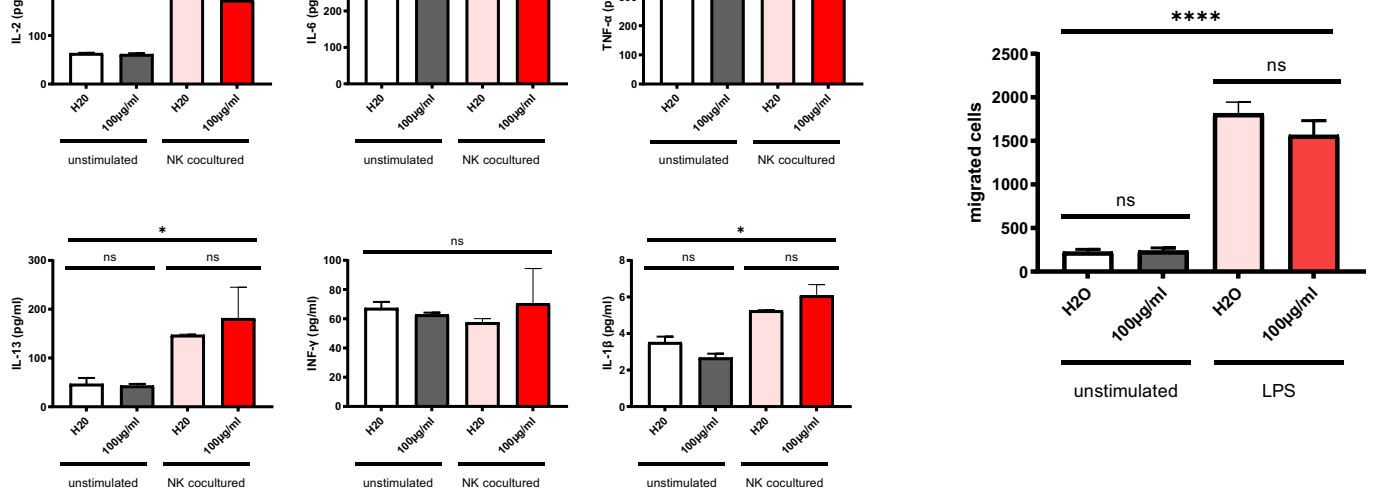

coats of healthy volunteers. DCs were generated from PBMCs in the presence of IL-4 and GM-CSF as previously described (Heine et al. 2013). First, we were able to show that immature DCs (iDCs) express relevant SLAMF7 on their surface (Fig. 1a). Toll-like receptor 4 (TLR4) stimulation and subsequent maturation even upregulated 
४Fig. 1 Elotuzumab spares the function of SLAMF7-positive dendritic cells. Human dendritic cells were generated in the presence of IL-4 and GM-CSF from peripheral blood mononuclear cells (PBMCs) harvested from buffy coats of healthy volunteer donors as described previously (Heine et al. 2013). Elotuzumab $(100 \mu \mathrm{g} / \mathrm{ml})$ was added every other day. LPS $(100 \mathrm{ng} / \mathrm{ml})$ was added on day 6 to mature iDCs, when indicated. NK cells were harvested from the same buffy coat by magnetic cell separation (NK cell isolation kit, Miltenyi Biotec, Bergisch Gladbach) and frozen until they were utilized. NK-DC cocultures (1:1 ratio) were performed with cells from the same buffy coat. DCs were harvested and analyzed after seven days of culture. All experiments were repeated at least three times with different donors. Representative donors are shown. a SLAMF7 expression was measured on immature (dark red) and mature DCs (light red). MFI and percentage expression of immature (dark red) and mature DCs (light red) are depicted. iDCs express SLAMF7, which is upregulated in $\mathrm{mDCs}(55.6 \%$ up to $96.18 \%)$. b CD1a expression of mature DC either treated with the vehicle $\mathrm{H}_{2} \mathrm{O}$ (dark red) or elotuzumab (100 $\mu \mathrm{g}$ / $\mathrm{ml}$ ) (light red) is unchanged, indicating that elotuzumab does not interfere with DC differentiation. c Viability of DCs generated from PBMCs in the presence of elotuzumab was measured via FACS. A viability above $80 \%$ shows no toxic effects of elotuzumab. d Expression of CD80, CD83 and CD86 is not inhibited by elotuzumab treatment. Elotuzumab-exposed NK cells induce expression of CD80 (70.36-84.99\%) an CD83 (7.37-15.79\%), but CD86 expression is slightly dampened (84.11-74.69\%). e Secretion of pro-inflammatory cytokines is not altered by elotuzumab pre-treatment. NK cells stimulate the secretion of IL-2, IL- $1 \beta$ or IL-13 by DCs, but elotuzumab does not potentiate this effect. f CCR7-dependent migration of DCs is not affected by elotuzumab in a transwell assay $(8 \mu \mathrm{m})$ towards a CCL19 gradient

SLAMF7 expression [ $55.6 \%$ on iDCs to $96.18 \%$ on mature DCs (mDCs)]. Next, we demonstrated that in vitro generation of DCs, measured by expression of the lineage marker CD1a, (Fig. 1b) and viability of human iDCs (Fig. 1c) is not affected by co-incubation with elotuzumab with concentrations up to $150 \mu \mathrm{g} / \mathrm{ml}$.

When elotuzumab was added during the differentiation period of naïve PBMC to DCs every other day (day 0, 2, 4 and 6) and iDCs were maturated by addition of lipopolysaccharide (LPS) on day 6, the expression of the co-stimulatory molecules CD40, CD80, CD83 and CD86 remained unaffected. iDCs co-incubated with NK cells alone or in the presence of elotuzumab for additional $24 \mathrm{~h}$ even showed a slight upregulation of CD80 (70.36-84.99\%) and CD83 (7.37-15.79\%. Expression of CD40 remained unaffected (99\%; data not shown), while CD86 (84.11-74.69\%) showed a slight downregulation (Fig. 1d). This indicates that activation markers of DCs are not significantly altered during Elotuzumab treatment.

It has already been shown in detail that binding of elotuzumab to SLAMF7 activates NK cells and that this enhances their interaction with macrophages (Campbell et al. 2018). Data regarding induction of cytokine production by binding of SLAMF7 on DCs or enhanced interaction of NK cells and DC by elotuzumab is lacking. Therefore, iDCs generated as described above were exposed to the SLAMF7 antibody elotuzumab alone or in the presence of NK cells. Interestingly, the presence of NK cells increased the secretion of IL-2, IL-13 and IL-1 $\beta$. Addition of elotuzumab had no effect on the respective cytokine release. Moreover, NK cells, elotuzumab or a combination of both did not affect cytokine levels of INF- $\gamma$, IL-6 and TNF $\alpha$.

Besides upregulation of activation markers and adequate cytokine production, migration of DC to lymphatic organs is a prerequisite for a robust immune response. Therefore, we investigated the migratory ability of mDCs, generated in the presence or absence of elotuzumab, in a transwell approach against a CCL19 gradient. Here, we could show that the CCR7-CCL19-dependent migration of DCs remains unaffected by elotuzumab (Fig. 1f).

Taken together, we could rule out direct suppressive effects of elotuzumab as well as indirect effects of elotuzumab-pre-treated NK cells on DC phenotype and function, such as cytokine production, chemokine-driven migration, induction of $\mathrm{T}$ cell responses and cytotoxicity.

Elotuzumab maintained the functionality of DCs, which is a prerequisite for robust immune responses to infectious pathogens as well as in the context of prophylactic vaccinations. However, the induction and duration of vaccination responses in MM patients has been described to be frequently decreased (Mustafa et al. 2019).Thus, the approach to prevent serious infectious complications by the application of prophylactic vaccinations may not be corrupted by SLAMF7 antibodies, although prior treatments might of course diminish immune responses. Our data shows that DC function is preserved, thus implicating that vaccination may be a feasible approach during elotuzumab treatment. Moreover, elotuzumab seems to be safe regarding promising novel therapeutic approaches for the treatment of MM containing vaccination regimes (Perumal et al. 2020).

In summary, we add insight into the immune shaping effects of MM treatment. Elotuzumab spares dendritic cell functionality and integrity but has previously been shown to stimulate NK cell function. Hence, elotuzumab might be a feasible option in MM patients at special risk for infections or the need for vaccination.

Author contributions All authors contributed to the study conception and design. LS, SD, SS and AH performed material preparation, data collection and analysis. SS, PB and AH wrote the first draft of the manuscript and all authors commented on previous versions of the manuscript. All authors read and approved the final manuscript.

Funding Open Access funding enabled and organized by Projekt DEAL. The EKFS Promotionskolleg "Neuroimmunology" funded this work (Grant number EKFS 2016-S3-02 to L.S.). Additionally, the Deutsche Krebshilfe (Mildred Scheel Nachwuchszentrum Grant; Grant number 70113307 to S.S.) and the Deutsche Forschungsgemeinschaft (DFG EXC2151—390873048 to A.H.) supported this research. 
Data availability Data is available upon request to the corresponding author.

\section{Compliance with ethical standards}

Conflict of interest The authors declare no competing financial interests.

Ethical approval The study was conducted in accordance with the Declaration of Helsinki and approval was obtained from the institutional ethics committee of the University of Bonn. (\#173/09).

Open Access This article is licensed under a Creative Commons Attribution 4.0 International License, which permits use, sharing, adaptation, distribution and reproduction in any medium or format, as long as you give appropriate credit to the original author(s) and the source, provide a link to the Creative Commons licence, and indicate if changes were made. The images or other third party material in this article are included in the article's Creative Commons licence, unless indicated otherwise in a credit line to the material. If material is not included in the article's Creative Commons licence and your intended use is not permitted by statutory regulation or exceeds the permitted use, you will need to obtain permission directly from the copyright holder. To view a copy of this licence, visit http://creativecommons.org/licenses/by/4.0/.

\section{References}

Campbell KS, Cohen AD, Pazina T (2018) Mechanisms of NK cell activation and clinical activity of the therapeutic SLAMF7 antibody, elotuzumab in multiple myeloma. Front Immunol 9:2551. https://doi.org/10.3389/fimmu.2018.02551

Carbone FR, Kurts C, Bennett SRM et al (1998) Cross-presentation: a general mechanism for CTL immunity and tolerance. Immunol Today 19:368-373. https://doi.org/10.1016/S0167-5699(98)01301 $-2$

Dimopoulos MA, Dytfeld D, Grosicki S et al (2018) Elotuzumab plus pomalidomide and dexamethasone for multiple myeloma. N Engl J Med. https://doi.org/10.1056/NEJMoa1805762

Dimopoulos MA, Lonial S, White D et al (2020) Elotuzumab, lenalidomide, and dexamethasone in RRMM: final overall survival results from the phase 3 randomized ELOQUENT-2 study. Blood Cancer J 10:91. https://doi.org/10.1038/s41408-020-00357-4

Girmenia C, Cavo M, Offidani M et al (2019) Management of infectious complications in multiple myeloma patients: expert panel consensus-based recommendations. Blood Rev 34:84-94. https ://doi.org/10.1016/j.blre.2019.01.001
Heine A, Held SAE, Daecke SN et al (2013) The JAK-inhibitor ruxolitinib impairs dendritic cell function in vitro and in vivo. Blood 122:1192-1202. https://doi.org/10.1182/blood-2013-03-484642

Hose D, Schreder M, Hefner J et al (2021) Elotuzumab, pomalidomide, and dexamethasone is a very well tolerated regimen associated with durable remission even in very advanced myeloma: a retrospective study from two academic centers. J Cancer Res Clin Oncol 147:205-212. https://doi.org/10.1007/s00432-020-03323-6

Hsi ED, Steinle R, Balasa B et al (2008) CS1, a potential new therapeutic antibody target for the treatment of multiple myeloma. Clin Cancer Res 14:2775-2784. https://doi.org/10.1158/1078-0432. CCR-07-4246

Mateos M-V, Dimopoulos MA, Cavo M et al (2018) Daratumumab plus bortezomib, melphalan, and prednisone for untreated myeloma. New England J Med 378:518-528. https://doi.org/10.1056/ NEJMoa1714678

Mustafa SS, Shah D, Bress J, Jamshed S (2019) Response to PCV13 vaccination in patients with multiple myeloma versus healthy controls. Human Vaccines Immunother 15:452-454. https://doi. org/10.1080/21645515.2018.1534516

Nencioni A, Garuti A, Schwarzenberg K et al (2006a) Proteasome inhibitor-induced apoptosis in human monocyte-derived dendritic cells. Eur J Immunol 36:681-689. https://doi.org/10.1002/ eji.200535298

Nencioni A, Schwarzenberg K, Brauer KM et al (2006b) Proteasome inhibitor bortezomib modulates TLR4-induced dendritic cell activation. Blood 108:551-558. https://doi.org/10.1182/blood -2005-08-3494

Perumal D, Imai N, Laganà A et al (2020) Mutation-derived Neoantigen-specific T-cell Responses in Multiple Myeloma. Clin Cancer Res: Off J Am Assoc Cancer Res 26:450-464. https://doi. org/10.1158/1078-0432.CCR-19-2309

Stocker N, Gaugler B, Ricard L et al (2020) Daratumumab prevents programmed death ligand-1 expression on antigen-presenting cells in de novo multiple myeloma. Cancer Med 9:2077-2084. https:// doi.org/10.1002/cam4.2827

Viola D, Dona A, Caserta E et al (2020) Daratumumab induces mechanisms of immune activation through CD38+ NK cell targeting. Leukemia. https://doi.org/10.1038/s41375-020-0810-4

Yamamoto K, Kitawaki T, Sugimoto N et al (2019) Anti-inflammatory modulation of human myeloid-derived dendritic cell subsets by lenalidomide. Immunol Lett 211:41-48. https://doi.org/10.1016/j. imlet.2019.05.012

Publisher's Note Springer Nature remains neutral with regard to jurisdictional claims in published maps and institutional affiliations. 